\title{
Glucocorticoid Exposure in Preterm Babies Predicts Saliva Cortisol Response to Immunization at 4 Months
}

\author{
VIVETTE GLOVER, RACHEL MILES, SIMON MATTA, NEENA MODI, AND JAMES STEVENSON \\ Wolfson and Weston Research Centre for Family Health [V.G., R.M., S.M.], Institute of Reproductive and \\ Developmental Biology, Imperial College London, London W12 ONN, UK; Faculty of Medicine [N.M.], \\ Imperial College London, Chelsea and Westminster Campus, London SW10 9NH, UK; Department of \\ Psychology [J.S.], Faculty of Medicine, Health and Life Sciences, and School of Psychology, University of \\ Southampton, Highfield, Southampton SO17 1BJ, UK
}

\begin{abstract}
ABSTR
Preterm babies are exposed to multiple stressors and this may
have long-term effects. In particular, high levels of endogenous
cortisol might have a programming effect on the hypothalamic-
pituitary-adrenal axis as may administered glucocorticoids. In
this study, we aimed to test the hypothesis that the level of
endogenous and exogenous glucocorticoid exposure during the
neonatal period predicts the saliva cortisol response to immuni-
zation at 4 mo of age. We followed 45 babies born below 32 wk
gestation. We showed that their concentration of plasma cortisol
during the first 4 wh was $358,314,231$, and 195 nmol/L cortisol,
respectively (geometric mean). This is four to seven times higher
than fetal levels at the same gestational age range. We used
routine immunization at 4 mo and 12 mo as a stressor and
measured the change in saliva cortisol as the stress response.
Mean circulating cortisol in the first 4 wk predicted the cortisol
\end{abstract}
There is considerable evidence from animal experiments that early exposure to stress, either in the antenatal or in the immediate postnatal periods, can have long term effects on both the function of the HPA axis, and on behavior (1-5). In general, the HPA axis of the offspring becomes hyperresponsive to a novel stressor. These effects are mediated, at least in part, by activation of the maternal HPA axis and the effect of fetal cortisol or corticosterone (in rodents) on the developing brain. These effects can, in some paradigms, be mimicked by synthetic glucocorticoids (6-8). Until recently, this has been little studied in humans. It does appear that cortisol from the mother crosses the placenta in sufficient amounts to affect the fetus $(9,10)$, although there is so far no

Received January 3, 2005; accepted May 272005.

Correspondence: Vivette Glover, D.Sc., Ph.D., Wolfson and Weston Research Centre for Family Health, Institute of Reproductive and Developmental Biology, Imperial College London, Du Cane Rd., London W12 0NN, UK; e-mail: v.glover@imperial.ac.uk

This study supported by Department of Health under the London, Research and Development, Responsive Funding Programme.

DOI: 10.1203/01.pdr.0000185132.38209.73 response at 4 but not at $12 \mathrm{mo}$. Path analysis showed that birthweight for gestational age, therapeutic antenatal steroids, and therapeutic postnatal steroids also contributed to the magnitude of the saliva cortisol response at 4 mo. This provides evidence that the magnitude of glucocorticoid exposure, both endogenous and exogenous, may have an effect on later stress responses. (Pediatr Res 58: 1233-1237, 2005)
Abbreviations
CFI, comparative fit index
CRH, corticotrophin releasing factor
HPA, hypothalamus-pituitary-adrenal
RMSEA, root mean square error of approximation
SDS, standard deviation score

direct evidence that maternal cortisol alters infant development. However, there is increasing evidence from epidemiologic studies that maternal stress and anxiety during pregnancy has an adverse effect on the development of the fetus and on the later behavior and cognitive development of the child (11-16).

Preterm babies are exposed both to a stressful environment, with concomitant endogenous glucocorticoid release, and may also be exposed, antenatally and postnatally, to exogenous glucocorticoids used therapeutically. If the animal models are relevant, one would predict that these infants would show long-term effects, both in HPA axis response and in behavior. Babies born extremely preterm are at risk of later behavioral problems, most noticeably with attention deficit disorders (17). Recent evidence suggests that antenatal glucocorticoid exposure has long-term effects on brain development (18). Davis et al. (19) have shown preterm babies exposed to antenatal betamethasone had a lower saliva cortisol response to a heel stick than matched controls at 3-6 d after delivery. $\mathrm{Ng}$ et al. (20) have also shown that one or two doses of antenatal 
corticosteroids were associated with a suppressed cortisol response to a CRH challenge at $\mathrm{d} 7$ but not $\mathrm{d} 14$. However, the longer-term effects of exogenous glucocorticoids and that of endogenously generated cortisol in the preterm infant have not been studied. The aim of this study was to determine the plasma level of cortisol in a cohort of preterm infants, and to determine whether the level of the endogenous and exogenous glucocorticoids during the neonatal period predicted subsequent cortisol responses to a standardized stressor.

To test the possible mechanisms underlying the association between endogenous and exogenous glucocorticoids in the perinatal period and later stress responses a path model was developed. This considered antenatal and postnatal steroids as separate possible influences on later stress responses, and also as possible influences on mean cortisol levels in the postnatal period. The sex of the child, gestational age, and birth weight were also considered as independent predictors of stress responses either directly or via their impact on postnatal glucocorticoids.

\section{METHODS}

Singleton infants born below 32 wk gestation without major immediate life-threatening congenital abnormalities, receiving care in the neonatal intensive care units at Hammersmith and Queen Charlotte's Hospitals, London, were eligible for recruitment into a trial examining the effects of mother-infant skin-to-skin contact on a range of infant and maternal outcomes. These hospitals were situated 5 miles apart in inner London and serve a multicultural urban population. They are under the management of a single National Health Service Healthcare Trust. The maternity and neonatal service is a referral center for high-risk patients. The neonatal service is provided by a single pediatric department and medical and nursing protocols for the two neonatal units are identical. The study was approved by the Research Ethics Committee of Imperial College Faculty of Medicine and Hammersmith Hospitals Trust and written maternal consent was obtained. The trial showed that there was no effect of the skin-to-skin intervention on plasma cortisol or later salivary cortisol baseline or response levels, or any other outcome measure and so data from all the infants were combined. The results of this trial are to be reported elsewhere.

Here we report data on circulating cortisol levels in the first 4 postnatal weeks, and salivary cortisol responses to the standardised stress of 4 and $12 \mathrm{mo}$ immunizations $(n=45)$. All subjects participated in these evaluations.

Illness severity. Preterm babies in intensive care have a variety of influences on their wellbeing. We used the number of days the infant received level 1 (full) intensive care according to predefined national criteria (21) as an index of illness severity.

Antenatal and postnatal steroids. Most of the mothers were given either betamethasone ( $n=31$; range, $1-16$ doses) or dexamethasone ( $n=5$; range, 1-4 doses) or both $(n=5)$ antenatally. Nine of the babies were given dexamethasone postnatally (range, 7-39 doses).

Blood samples. Basal plasma cortisol level during wk 1-4 post birth was measured in a once weekly $0.5-\mathrm{mL}$ blood sample and in residual plasma volumes left over from samples taken at routine clinical samples. These samples could have been obtained on any day of the specified week. If more than one sample was obtained during a particular week, as was generally the case, the results were meaned. Blood samples were collected in heparinized vacutainers, and spun at $3000 \mathrm{~g}$ for $15 \mathrm{~min}$. The plasma aliquot was removed and frozen at $-80^{\circ} \mathrm{C}$ in polypropylene storage vials until assay.

Saliva samples. Infants were recalled to hospital to receive routine 4- and 12-mo immunizations in accordance with the UK national schedule. Immunizations were administered at standard times (between 1200 and $1600 \mathrm{~h}$ at $4 \mathrm{mo}$; between 1000 and $1200 \mathrm{~h}$ at $12 \mathrm{mo}$ ) to control for possible diurnal variation. Saliva samples were obtained immediately before and $20 \mathrm{~min}$ after injection (the time taken for cortisol to rise after acute stress). Fruit juices, sweet drinks and milk feeds were avoided for $30 \mathrm{~min}$ before immunization. Saliva was collected into salivettes and stored frozen until assay

Cortisol assays. These were carried out using Coat-A-Count DPC RIA (Diagnostic Products, Los Angeles, CA).

Data analyses. Each variable was tested for the normality of its distribution. Mean plasma cortisol wk 1-4 was log transformed to achieve a normal distribution for statistical analyses. (Values in the text are given in geometric means to show actual concentrations.) Univariate and multivariate correlations were initially sought between the level of endogenous cortisol during the neonatal period and the pre minus post immunization salivary cortisol responses ( $\Delta$ cortisol) to 4 - and 12 -mo immunization. Path analysis (22) was used to test a model of the putative causal relationships between factors influencing $\Delta$ cortisol at $4 \mathrm{mo}$. The final model reported is that which retains only significant paths. Its fit to the data (the measured covariances between the variables) is estimated using $\chi^{2}$, comparative fit index (CFI), and root mean square error of approximation (RMSEA). A model shows good fit if $\chi^{2}$ is not significant, the CFI is $>0.90$ and the RMSEA is $<0.08$ (22).

\section{RESULTS}

Infant ages for the first immunization assessment were chronological age $21.7 \pm 4.0 \mathrm{wk}$ (mean $\pm \mathrm{SD}$ ), corrected age 9.4 $\pm 4.4 \mathrm{wk}$; and for the second assessment, chronological age $55.3 \pm 5.6$, corrected age $42.8 \pm 4.1 \mathrm{wk}$.

Figure 1 shows the individual infant plasma cortisol levels during wk 1-4. The geometric means for plasma cortisol during the first $4 \mathrm{wk}$ were 358, 314, 231, and $195 \mathrm{nmol} / \mathrm{L}$, respectively.

Cortisol responses to inoculation at 4 mo were available on 45 infants. Infant characteristics are shown in Table 1. Although there was a clear saliva cortisol response to inoculation at 4 mo ( $p<0.001$ paired $t$ test), the mean response was much reduced by 12 mo ( $p=0.006$ paired $t$ test for deltas at the two time points).

Most of these babies spent some of the first 4 wk in full level 1 intensive care (Table 1). Although there was no correlation between the time spent and mean plasma cortisol weeks 1-4 in the whole group, there was a significant difference between those who spent less than a week $(n=10)$, geometric mean, $243 \mathrm{nmol} / \mathrm{L}$, and the rest, $335 \mathrm{nmol} / \mathrm{L}(p=0.014)$.

An initial correlation analysis (Table 2) showed that the saliva cortisol response to inoculation at 4 mo was significantly correlated with both the mean plasma cortisol in the first $4 \mathrm{wk}$ and to birthweight SDS (Child Growth Foundation, London, UK). There was no correlation between baseline saliva cortisol at 4 mo and mean plasma cortisol wk 1-4. Also, there was no significant correlation between baseline saliva cortisols at 4 and 12 mo or between the $\Delta$ cortisols at the two time points. There was a significant positive association between antenatal steroids and baseline cortisol at 4 mo. The correlation patterns were similar whether total maternal antenatal steroid exposure

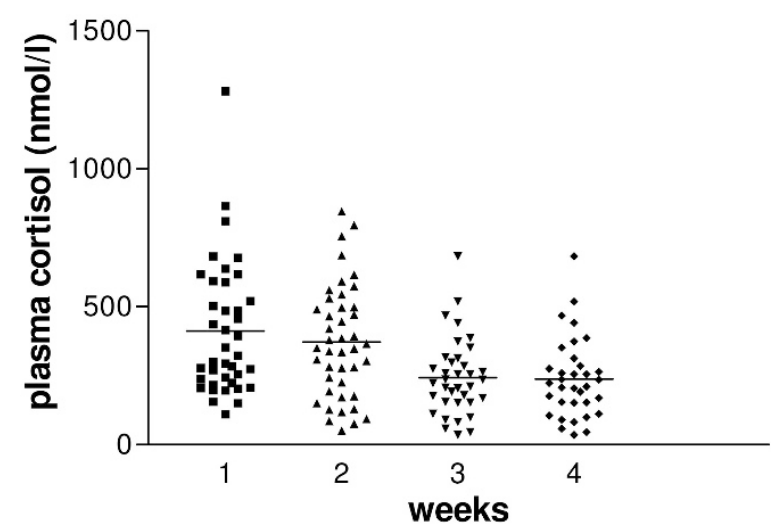

Figure 1. Mean plasma cortisol for each baby in the study $(n=45)$ for each of the first $4 \mathrm{wk}$. 
Table 1. Characteristics of babies in the study and their saliva cortisol values $(n=45)$

\begin{tabular}{lc}
\hline & $\begin{array}{c}\text { Mean } \pm \text { SD or } \\
\text { median (range) of } \\
\text { babies }(n=45)\end{array}$ \\
\hline Sex, M/F & $25 / 20$ \\
Gestational age at birth (wk) & $28.0 \pm 2.2$ \\
Birth weight (g) & $1095 \pm 424$ \\
Type of delivery, vaginal/cesarean & $20 / 25$ \\
Number of doses of surfactant given & $1(1-3)$ \\
Number of days receiving full (level 1) & $12(0-90)$ \\
$\quad$ intensive care weeks 1-4 & $4.5 \pm 2.8$ \\
Baseline saliva cortisol at 4 mo (nmol/L) & $4.0 \pm 6.8$ \\
Delta saliva cortisol at 4 mo (nmol/L) & $5.5 \pm 9.0$ \\
Baseline saliva cortisol at 12 mo (nmol/L) & $-0.2 \pm 5.6$ \\
Delta saliva cortisol at 12 mo (nmol/L) & \\
\hline
\end{tabular}

or dexamethasone or betamethasone were used separately. There was no correlation between antenatal and postnatal exogenous steroid exposure.

A multivariate analysis (Table 3) confirmed the independent contribution of birth weight SDS and mean plasma cortisol wk 1-4 to $\Delta$ cortisol at $4 \mathrm{mo}$, but also showed an additional negative association with therapeutic antenatal maternal steroids. This analysis showed that nearly half the variance in $\Delta$ cortisol can be predicted by these three factors $\left(R^{2}=0.46\right)$. Males tended to have a larger response (see Table 3).

A path model was developed where the paths between variables were based on their relationships over time (Fig. 2). The paths retained are all significant and there are no additional significance factors. This model fits the data well $\left(\chi^{2}=16.34\right.$, $\mathrm{df}=14$, NS; CFI $=0.934$; RMSEA $=0.062\left(\mathrm{CI}_{.90} 0.000-\right.$ $0.163)$. The model shows that the postnatal steroids have a dual contribution to $\Delta$ cortisol, a direct positive predictive effect together with a negative effect on mean cortisol wk 1-4. This might explain why their effect was missed in the previous analyses. The path analysis confirms that antenatal steroids, postnatal steroids, plasma cortisol wk 1-4, birth weight SDS, and gestational age all independently predicted $\Delta$ cortisol at 4 mo.

\section{DISCUSSION}

This study confirms that preterm babies are exposed to high levels of endogenous cortisol (23). The levels found here were four to seven times higher than that documented in fetuses at equivalent gestational ages $(9,10)$. Given the extensive evidence from animal experiments $(5,6)$, it is a plausible hypothesis that this may have long-term effects on the human HPA axis.

It is well recognized that some preterm babies manifest a hypoadrenal response (24). However, none of the infants in this study showed any overt manifestation suggestive of this, such as intractable hypotension responsive only to glucocorticoid therapy. We did find a wide range of basal cortisol values (from 110 to $1282 \mathrm{nmol} / \mathrm{L}$ in wk 1 , and from 35 to $683 \mathrm{nmol} / \mathrm{L}$ in wk 4). There was also a wide range of cortisol responses to inoculation at $4 \mathrm{mo}$ (from -11.1 to $27.9 \mathrm{nmol} / \mathrm{L}$ ).

We used both blood and saliva for cortisol assays. Other studies have shown good correlation between plasma and saliva cortisol levels (25). Although the blood samples were collected at varying times of day, preterm infants of this postnatal age, unlike adults, do not exhibit diurnal variation in cortisol production (26). The nature of the method for blood collection (needle or indwelling catheter) would not be expected to have any effect on cortisol levels as the cortisol response to an invasive procedure takes at least $10-20 \mathrm{~min}$ to develop.

The results presented here show that there is a positive association between the endogenous cortisol response during the first $4 \mathrm{wk}$ and the infant saliva cortisol response to a stressor 4 mo later. The effect of postnatal steroids was similar to that of the postnatal endogenous cortisol. However, the effects shown in the path analysis are complex; antenatal steroids had a negative correlation with the infants' saliva cortisol response at 4 mo. The negative relationship between antenatal steroids and the saliva cortisol response of preterm babies is similar to that reported in other studies with infants studied in the first week $(19,20)$. Antenatal steroids were also associated with a raised baseline cortisol level at 4 mo, which was in turn link with a reduced response to stress (Table 2). Lower birth weight SDS was also associated with a reduced response to immunization at $4 \mathrm{mo}$. Low birth weight for gestational age can be indicative of fetal stress and also be associated with higher endogenous fetal glucocorticoid levels (27). The effect of antenatal therapeutic steroids thus has some similarities to high antenatal endogenous levels (as indicated by low birth weight for gestation), and postnatal therapeutic steroids appear similar in effect to postnatal endogenous cortisol. Why responses to antenatal and postnatal exposure should differ is not clear and needs further confirmation and exploration. It is unlikely to be due solely to maturational stage, given the overlap in gestational age at which antenatal and postnatal exposure occurred. It presumably reflects other differences in the intrauterine and postnatal environmental milieu.

Grunau et al. (28) have shown that basal saliva cortisol at 8 mo corrected age, was higher in babies born at $<29$ wk compared with those born at 29-32 wk or full term. In the current study no association was found between plasma cortisol levels in the first $4 \mathrm{wk}$ and basal cortisol levels at 4 mo or 12 mo. However, there was a nonsignificant trend for those born before $29 \mathrm{wk}$ to have higher basal cortisol levels at $12 \mathrm{mo}$ than those born later.

This cohort of infants had, in general, a substantially diminished saliva cortisol response to immunization by $12 \mathrm{mo}$ (Table 1). In this they resembled full-term babies, in whom this phenomenon of a reduction in saliva cortisol response by one year is well established (29). The reason for this change in responsiveness of the HPA axis in the first year is still not clear. It does not mean that there is no longer-term programming of the HPA axis in humans, but that it may be complex, and will need different methods of investigation from that in younger babies.

It is of interest that preterm infants have a similar spectrum of developmental and behavioral problems to babies whose mothers have been very stressed or anxious antenatally. Both show increased levels of attention deficit and hyperactivity, 
Table 2. Univariate correlation coefficients between predictors and $\Delta$ cortisol and baseline cortisol

\begin{tabular}{|c|c|c|c|c|c|c|c|c|c|c|}
\hline Variables & Sex & $\begin{array}{c}\text { Antenatal } \\
\text { steroids }\end{array}$ & $\begin{array}{l}\text { Gestational } \\
\text { age }\end{array}$ & $\begin{array}{l}\text { Birth weight/ } \\
\text { gestational age } \$\end{array}$ & $\begin{array}{c}\text { Postnatal } \\
\text { steroids }\end{array}$ & $\begin{array}{c}\text { Mean log } \\
\text { cortisol } \\
\text { wk 1-4 }\end{array}$ & $\begin{array}{c}\text { Baseline } \\
\text { cortisol } \\
\text { at } 4 / 12\end{array}$ & $\begin{array}{c}\Delta \text { Cortisol } \\
\text { at } 4 / 12\end{array}$ & $\begin{array}{c}\text { Baseline } \\
\text { cortisol } \\
\text { at } 12 / 12\end{array}$ & $\begin{array}{c}\Delta \text { Cortisol } \\
\text { at } 12 / 12\end{array}$ \\
\hline Sex & 1.00 & -0.118 & -0.028 & 0.028 & $-416^{* *}$ & $0.364^{*}$ & 0.040 & -0.123 & 0.162 & -0.113 \\
\hline Gestational age & & & 1.00 & 0.090 & -0.226 & -0.103 & 0.063 & 0.230 & -0.121 & 0.131 \\
\hline Birth weight/gestational age $\mathrm{a}^{\S}$ & & & & 1.00 & -0.187 & 0.074 & -0.076 & $0.365^{*}$ & -0.046 & -0.028 \\
\hline Postnatal steroids & & & & & 1.00 & $-0.478 * *$ & -0.159 & -0.044 & -0.108 & 0.075 \\
\hline$\Delta$ Cortisol at $4 / 12$ & & & & & & & & 1.0 & -0.128 & 0.112 \\
\hline Baseline cortisol at $12 / 12$ & & & & & & & & & 1.0 & $-0.757 * *$ \\
\hline$\Delta$ Cortisol at $12 / 12$ & & & & & & & & & & 1.0 \\
\hline
\end{tabular}

$* p<0.05 ; * * p<0.01$. Calculated as a SDS.

Table 3. Simultaneous entry of predictors of $\Delta$ cortisol at 4 mo

\begin{tabular}{lcc} 
& $\left(R^{2}=45 \%\right)$ & \\
\hline \multicolumn{1}{c}{ Predictor variables } & $\beta$ & $p$ \\
\hline Sex & -0.264 & 0.055 \\
Antenatal steroids & -0.263 & 0.038 \\
Gestational age & 0.351 & 0.010 \\
Birth weight SDS & 0.334 & 0.009 \\
Postnatal steroids & 0.218 & 0.155 \\
Mean ln cortisol wk 1-4 & 0.529 & 0.001 \\
\hline
\end{tabular}

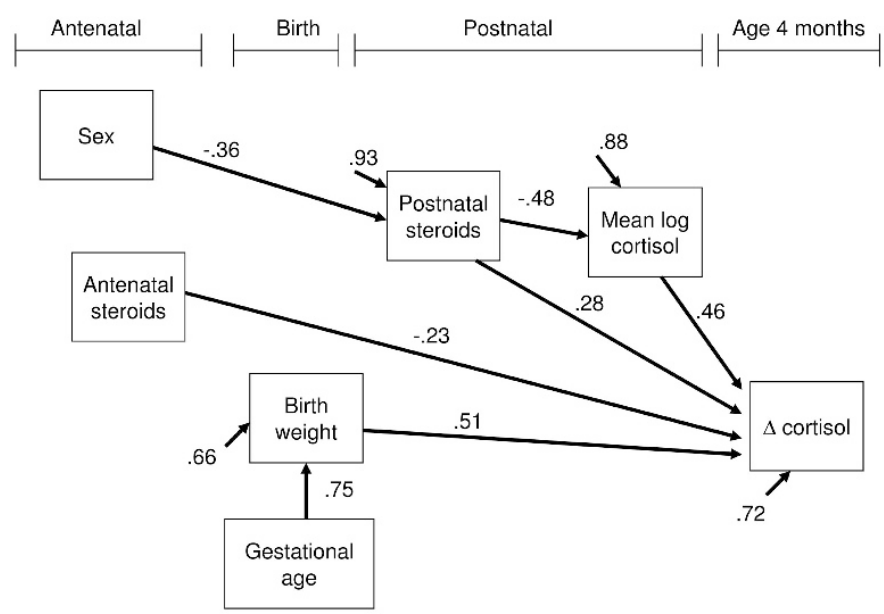

Figure 2. Path analysis showing significant determinants of the $\Delta$ cortisol response to inoculation at 4 mo. Birth weight indicates birth weight SDS

anxiety and depression $(14,17)$. We have suggested that the long-term effects of preterm stress or anxiety may in part be due, as in the animal models, to exposure of the fetus to increased cortisol (30). Although the most sensitive period to gestational stress in humans is not established, it has been suggested that maternal anxiety at $32 \mathrm{wk}$ has a greater effect than maternal anxiety at $18 \mathrm{wk}(14)$. Thus, exposure in the last trimester of pregnancy may be important, and would coincide with the period of exposure to raised cortisol of preterm infants.

There are obviously multiple potential influences on HPA reactivity in the perinatal period. Our use of path analysis is an attempt to tease out those that are most relevant. The programming hypothesis is strengthened by the effect of postnatal steroids given to the infant in the first $4 \mathrm{wk}$. These had the expected effect of reducing the plasma cortisol level at the time, but also having a direct independent positive effect on the later response.

Several caveats must be applied to this study. The numbers were small. Preterm babies are a heterogeneous group with many underlying co-morbidities that may affect outcomes. Both antenatal and postnatal exogenous steroids are given for specific reasons, and these could have independent effects on the development of HPA axis responses. Other antenatal stressors to the mother or postnatal to the infant could have independent effects. Variable levels of placental $11 \beta$ hydroxysteroid dehydrogenase may alter fetal exposure to glucocorticoids. It was not possible to resolve these issues in this study

It is possible that the more reactive infants showed a higher response both early and later. However, given the different degrees of stressful procedures that different infants are exposed to, it seems unlikely that the main determinant of the early plasma cortisol level is genetic. The findings with the effects of the postnatal exogenous glucocorticoids also support a programming hypothesis.

In conclusion, the results presented here suggest that the exposure to high levels of endogenous and exogenous cortisol, experienced by many preterm infants, may have an effect on the later function of their HPA axis. These questions need to be explored further in larger cohorts. Even if these effects only last for the time demonstrated here, up to 4 mo, they could be important for behavioral and other development, including the immune response (31).

Acknowledgments. The authors thank Diana Adams for very able assistance, participating babies and their parents, and the nurses on the neonatal intensive care units.

\section{REFERENCES}

1. Barbazanges A, Piazza PV, Le Moal M, Maccari S 1996 Maternal glucocorticoid secretion mediates long-term effects of prenatal stress. J Neurosci 16:3943-3949

2. Clarke AS, Wittwer DJ, Abbott DH, Schneider ML 1994 Long-term effects of prenatal stress on HPA axis activity in juvenile rhesus monkeys. Dev Psychobiol 27:257-69

3. Henry C, Kabbaj M, Simon H, Le Moal M, Maccari S 1994 Prenatal stress increases the hypothalamo-pituitary-adrenal axis response in young and adult rats. J Neuroendocrinol 6:341-345

4. Uno H, Lohmiller L, Thieme C, Kemnitz JW, Engle MJ, Roecker EB, Farrell PM 1990 Brain damage induced by prenatal exposure to dexamethasone in fetal rhesus macaques. I. Hippocampus Brain Res Dev Brain Res 53:157-67

5. Weinstock M 2001 Alterations induced by gestational stress in brain morphology and behavior of the offspring. Progr Neurobiol 65:427-451 
6. O'Regan D, Welberg LL, Holmes MC, Seckl JR 2001 Glucocorticoid programming of pituitary-adrenal function: mechanisms and physiological consequences. Semin Neonatol 6:319-329

7. Matthews SG, Owen D, Banjanin S, Andrews MH 2002 Glucocorticoids, hypothalamo-pituitary-adrenal (HPA) development, and life after birth. Endocr Res 28:709-18

8. Banjanin S, Kapoor A, Matthews SG 2004 Prenatal glucocorticoid exposure alters hypothalamic-pituitary-adrenal function and blood pressure in mature male guinea pigs. J Physiol 558:305-318

9. Gitau R, Cameron A, Fisk NM, Glover V 1998 Fetal exposure to maternal cortisol Lancet 352:707-8

10. Gitau R, Fisk NM, Cameron A, Teixeira JM, Glover V 2001 Fetal hypothalamicpituitary-adrenal stress responses to invasive procedures are independent of maternal responses. J Clin Endocrinol Metab 86:104-109

11. Lou HC, Hansen D, Nordentoft M, Pryds O, Jensen F, Nim J, Hemmingsen R 1994 Prenatal stressors of human life affect fetal brain development. Dev Med Child Neurol 36:826-832

12. Brouwers EP, van Baar AL, Pop VJ 2001 Maternal anxiety during pregnancy and subsequent infant development. Inf Behav Develop 24:95-106

13. Huizink AC, de Medina PG, Mulder EJ, Visser GH, Buitelaar JK 2002 Psychologica measures of prenatal stress as predictors of infant temperament. J Am Acad Child Adolesc Psychiatry 41:1078-1085

14. O'Connor TG, Heron J, Golding J, Beveridge M, Glover V 2002 Maternal antenata anxiety and children's behavioural/emotional problems at 4 years. Br J Psychiatry 180:502-508

15. O'Connor TG, Heron J, Glover V Alspac Study Team 2002 Antenatal anxiety predicts child behavioral/emotional problems independently of postnatal depression. J Am Acad Child Adolesc Psychiatry 41:1470-1477

16. Laplante DP, Barr RG, Brunet A, Galbaud du Fort G, Meaney ML, Saucier JF, Zelano PR, King S 2004 Stress during pregnancy affects general intellectual and language functioning in human toddlers. Pediatr Res 56:400-410

17. Hille ET, den Ouden AL, Saigal S, Wolke D, Lambert M, Whitaker A, Pinto-Martin JA, Hoult L, Meyer R, Feldman JF, Verloove-Vanhorick SP, Paneth N 2001 Behavioural problems in children who weigh $1000 \mathrm{~g}$ or less at birth in four countries. Lancet 357:1641-1643
18. Modi N, Lewis H, Al-Naqeeb N, Ajayi-Obe M, Doré CJ, Rutherford M 2001 The effects of repeated antenatal glucocorticoid therapy on the developing brain. Pediatr Res 50:581-585

19. Davis EP, Townsend EL, Gunnar MR, Georgieff MK, Guiang SF, Ciffuentes RF, Lussky RC 2004 Effects of prenatal betamethasone exposure on regulation of stress physiology in healthy premature infants. Psychoneuroendocrinology 29:1028-1036

20. Ng PC, Lam CW, Lee CH, Ma KC, Fok TF, Chan IH, Wong E 2002 Reference ranges and factors affecting the human corticotropin-releasing hormone test in preterm, very low birth weight infants. J Clin Endocrinol Metab 87:4621-4628

21. British Association of Perinatal Medicine 1992 Standards for hospitals providing neonatal intensive and high dependency care and categories of babies requiring neonatal care. British Association of Perinatal Medicine, London

22. MacCallum RC, Austin JT 2000 Applications of structural equation modeling in psychological research. Annu Rev Psychol 51:201-226

23. Wittekind CA, Arnold JD, Leslie GI, Luttrell B, Jones MP 1993 Longitudinal study of plasma ACTH and cortisol in very low birth weight infants in the first 8 weeks of life. Early Hum Dev 33:191-200

24. Seri I, Tan R, Evans J 2001 Cardiovascular effects of hydrocortisone in preterm infants with pressor-resistant hypotension. Pediatrics 107:1070-1074

25. Calixto C, Martinez FE, Jorge SM, Moreira AC, Martinelli CE 2002 Correlation between plasma and salivary cortisol levels in preterm infants. J Pediatr 140:116-118

26. de Weerth C, Zijl RH, Buitelaar JK 2003 Development of cortisol circadian rhythm in infancy. Early Hum Dev 73:39-52

27. Economides DL, Nicolaides KH, Campbell S 1991 Metabolic and endocrine findings in appropriate and small for gestational age fetuses. J Perinat Med 19:97-105

28. Grunau RE, Weinberg J, Whitfield MF 2004 Neonatal procedural pain and preterm infant cortisol response to novelty at 8 months. Pediatrics 114:77-84

29. Gunnar MR, Donzella B 2002 Social regulation of the cortisol levels in early human development. Psychoneuroendocrinology 27:199-220

30. Glover V, O'Connor TG 2002 Effects of antenatal stress and anxiety: implications for development and psychiatry. Br J Psychiatry 180:389-391

31. Leonard B 2000 Stress, depression and the activation of the immune system. World J Biol Psychiatry 1:17-25 